\title{
Lusotopie
}

Recherches politiques internationales sur les espaces

issus de l'histoire et de la colonisation portugaises

$\mathrm{XV}(1) \mid 2008$

Indiens du Mozambique et d'Afrique orientale

\section{Africana Studia. Revista internacional de estudos africanos}

Porto, Centro de estudos africanos da Universidade do Porto, 8, 2005, $340 \mathrm{p}$.

\section{Michel Cahen}

\section{(2) OpenEdition}

Journals

Édition électronique

URL : http://journals.openedition.org/lusotopie/888

ISSN : $1768-3084$

Éditeur :

Association des rechercheurs de la revue Lusotopie, Brill, Karthala

Édition imprimée

Date de publication : 30 juin 2008

Pagination : 245-249

ISSN : 1257-0273

Référence électronique

Michel Cahen, «Africana Studia. Revista internacional de estudos africanos », Lusotopie [En ligne], XV

(1) | 2008, mis en ligne le 10 mars 2016, consulté le 20 avril 2019. URL : http://

journals.openedition.org/lusotopie/888

Ce document a été généré automatiquement le 20 avril 2019

Tous droits réservés 


\title{
Africana Studia. Revista internacional de estudos africanos
}

Porto, Centro de estudos africanos da Universidade do Porto, 8, 2005, $340 \mathrm{p}$.

\author{
Michel Cahen
}

\section{RÉFÉRENCE}

Africana Studia. Revista internacional de estudos africanos (Porto, Centro de estudos africanos da Universidade do Porto), 8, 2005, 340 p., ISSN : 0874-2375.

1 Cette livraison contient en particulier un dossier «Os Estados lusófonos em África, 1975-2005»(p. 9-163). Le Mozambique y est surreprésenté (4 des 7 articles), ce qui confirme une fois de plus la «supériorité » de ce pays dans l'historiographie des Palop. Citons :

2 - Franz HEIMER, Elisete Marques da SILVA \& Gabriel Mithá RIBEIRo, «Representações sociais, valores e atitudes face ao político em Angola e Moçambique» (p. 11-38). C'est le seul article qui aborde réellement l'Angola. Il s'agit d'approcher la culture politique populaire en Angola et au Mozambique par le biais de questionnaires - ce qui pose tous les problèmes de l'usage des méthodes quantitatives pour approcher un diagnostic éminemment qualitatif dans des pays où le rapport à l'État est problématique.

3 - Michel CAHEN, "Luta de emancipação anticolonial ou movimemto de libertação nacional ? Processo histórico e discurso ideológico - o caso das colónias portuguesas e de Moçambique em particular» (p. 39-67), est la version en langue portugaise d'un article précédement publié en français dans la Revue Historique (Paris, PUF), janvier 2006. À signaler quelques erreurs de traduction (notamment ligne 2 p. 60 où «muito semelhantes » doit être lu «muito diferentes»).

4 - Felizardo BovenE, «Moçambique, 30 anos de independência » (p. 69-84), est un article dont on comprend mal l'insertion dans ce volume. 
5 - Rita PAís, «A terceira vitória da Frelimo em Moçambique : marginalização política ou estabilidade económica?»(p.85-98), revient sur la troisième victoire électorale du Frelimo au Mozambique (2004). Il est dommage que l'auteur considère comme fiable les données publiées par le pouvoir. Si elle a raison de souligner la faiblesse organisationnelle et politique de la Renamo, elle ne se pose aucune question sur les raisons du massif «abstentionnisme» dans les zones rurales pro-Renamo, dû en grande partie à un sabotage du recensement et aux inversions de listes électorales, bien avant le scrutin...

6 - Daniel A. PEREIRA, «Cabo Verde, Trinta anos de independência nacional » (p. 99-108); l'auteur est un proche collaborateur du gouvernement. Son article met surtout l'accent sur le rôle de la diplomatie de l'archipel, ce qui laisse sur sa fin.

7 - Leopoldo AMADO, «Guiné-Bissau: 30 anos de independência » (p. 109-135); il est dommage que l'auteur ait cru bon de revenir dans cet article jusqu'au début du siècle, ce qui n'apporte rien de nouveau. En revanche, la fin de son article est beaucoup plus approfondie (période 1994-1998/2005).

8 - Gerhard SEIBERT, «O semi-presidencialismo e o contrôle da constitucionalidade em São Tomé e Prícipe » (p.137-163), est une intéressante étude sur le pouvoir à São Tomé, et notamment sur les éternels conflits entre le Président et la majorité parlementaire (ici deux cas sont étudiés: l'exonération du Premier ministre par le Président, et l'établissement de relations diplomatiques contre la volonté de l'Assemblée - ici le cas de Taïwan versus la R. P. de Chine).

9 Ce dossier regroupe donc des textes de valeurs inégales, mais souligne aussi la difficulté à faire vivre une revue internationale, de langue principalement portugaise, hors de la capitale dans un pays hyper-centralisé...

Hors dossier, il faut noter :

10 - Eduardo Costa DIAS, " "Islão Negro" versus "verdadeiro Islão". Dilema ou sintomas de persistente e injustificada discriminação dos Muçulmanos africanos subsarianos?» (p. 165-187). Cet article présente une version fouillée, complexe et nuancée de l'ancienne question relative à la caratérisation de l'islam en Afrique subsaharienne. L'auteur, avec d'autres, récuse l'idée de l'islam «noir » tout en soulignant les disciminations sociales fondatrices au sein de ces communautés musulmanes d'Afrique subsaharienne, surtout quand sur le même espace vivent des communaiutés d'origine ethniques différentes (Arabes, Ouest-Africains, etc.).

Le volume contient encore une section de critique de livres portant sur l'esclavage. Il vaut la peine de consacrer un long développement à l'un des comptes rendus :

11 - João Pedro MARQUeS, «Foram os escravos decisivos na abolição da escravidão? Considerações a propósito de um livro de Nelly Schmidt» (p. 231-257). Loin d'être une simple recension, ce texte est un article de critique vitriolique d'un récent livre de Nelly Schmidt (L'abolition de l'esclavage. Cinq siècles de combats ( $\mathrm{XI}^{e}-\mathrm{XX}^{e}$ siècles), Paris, Fayard, 2005, 450 p., ISBN-13 : 978-2-213-62222-4). On peut partir de l'hypothèse que J.P. Marques a raison sur une partie des points qu'il soulève. Le seul titre de l'ouvrage de Schmidt attire en effet la suspicion ( «Cinq siècles de combats »), qui rappelle le mythe parallèle (et donc équivalent) que les nationalistes africains ont opposé au mythe portugais des " cinq siècles de colonisation", à savoir leurs "cinq siècles de résistance ». Or, bien évidemment, toutes les guerres entre des États africains et le Portugal (ou autre puissance coloniale) n'ont pas été, côté africain, des «luttes anticoloniales », mais de classiques guerres d'État à État. Toute résilience n'est pas résistance ouverte, et tout acte de 
résistance ouverte n'est pas forcément antisystémique (ici donc, anti-esclavagiste). J.P. Marques a donc certainement raison de constater que maintes révoltes d'esclaves visèrent à libérer un groupe bien défini d'entre eux, mais nullement à supprimer l'esclavagisme (même les Quilombos firent des esclaves, même Toussaint eut les siens). Par ailleurs, il ne suffit pas d'égrener des listes de révoltes, évidemment souvent mal documentées, pour prouver que ce sont ces révoltes d'esclaves qui ont été le fer de lance poussant à l'abolitionnisme en Europe. Haïti (1804) apparait comme cas unique et ne mena pas la France à abolir avant 1848 .

12 Cependant, le propos de J.P. Marques paraît outrancier, dans le ton ${ }^{1}$ comme sur le fond. Pour exprimer ses désaccords avec Schmidt, Marques qualifie les thèses défendues par cette dernière de "manipulation ", "subterfuge », etc. Mais il ne pose jamais la question - quelle que soit la réponse qu'il faut lui donner - de savoir si l'historiographie a pu sousestimer le poids des luttes sociales serviles dans le progrès de l'abolitionnisme. Même si la conclusion devait être négative, il n'est pas scandaleux de se la poser. Porté par sa fougue, J.P. Marques dit aussi des choses fausses à l'appui de sa critique de Schmidt. La loi Taubira, en France n'a pas imposé un contenu officiel aux programmes scolaires, à la différence de la proposition de loi de février 2005 sur les «aspects positifs de la colonisation $\star^{2}$ : elle a simplement voulu garantir que l'histoire de la traite y serait présente $^{3}$; elle n'a pas qualifié l'esclavage de "génocide», mais de "crime contre l'humanité »; elle a institué une journée du Souvenir ${ }^{4}$. On aimerait que le Portugal, qui organisa en 1998 à Lisbonne l'Exposition universelle sur le thème des "Océans", dans laquelle n'exista nul pavillon de la traite, en fit autant. Certes, il serait aberrant de considérer les trafiquants comme des « coupables » et Voltaire ou Montesquieu comme des « complices »- du fait qu'ils n'ont pas dénoncé suffisamment la traite - de ce crime contre l'humanité, s'agissant de faits survenus à une autre époque qui avait d'autres valeurs. Mais pourquoi ne pas dire à ceux qui se considèrent «descendants d'esclaves ", et à tout le monde, que nous considérons bien, aujourd'hui, que ce trafic fut un crime contre l'humanité ? Les Jeunes Turcs ottomans qui, à une autre époque (1915) et avec d'autres valeurs, massacrèrent les Arméniens, n'ont pas eu, de leur côté, le sentiment de commettre un crime contre l'humanité (et encore moins un génocide). Cela inviabilise-t-il la revendication actuelle des Arméniens pour la reconnaissance de ce génocide?

13 Enfin, J.P. Marques établit une relation malheureuse avec la plainte déposée contre O. Pétré-Grenouilleau. Or la plainte contre cet historien français - du reste retirée rapidement par ses promoteurs - n'a pas été déposée contre son livre (ce que semble croire J.P. Marques) $)^{5}$, mais contre des propos tenus par lui dans la presse grand public. Or ses propos furent fort malheureux, comme on peut en juger :

«... la traite, c'est-à-dire le commerce des esclaves, n'est apparue qu'au vII siècle, vers 650. C'est l'empire musulman [sic] qui a commencé la traite. Pour une raison simple : les musulmans n'ont pas le droit d'avoir des esclaves musulmans. Ils [sic] se sont donc tournés vers l'Europe et vers l'Afrique noire pour acheter ces esclaves. La traite négrière s'est achevée vers 1920. [...] On sait que l'Afrique noire [sic] a été victime et acteur de la traite. Les historiens, quelles que soient leurs convictions politiques, sont d'accord là dessus. [...] C'est aussi le problème [sic] de la loi Taubira qui considère la traite des Noirs par les Européens comme un "crime contre l'humanité", incluant de ce fait [sic] une comparaison avec la Shoah. Les traites négrières ne sont pas des génocides. La traite n'avait pas pour but d'exterminer un peuple. L'esclave était un bien qui avait une valeur marchande qu'on voulait faire travailler le plus possible. ${ }^{6}$ 

« ottoman » ou « moghol » : aurait-on dit que la traite atlantique fut le fruit de l'« empire chrétien »?), des généralisations abusives sur les musulmans («ils se sont tournés vers l'Europe et l'Afrique noire ») et les Africains (ce serait l'« Afrique noire », et non certains États africains, qui aurait été l'un des acteurs de la traite), l'argumentation de l'entrevue (plus encore que de l'ouvrage) écartait la spécificité de la traite européenne. Or si le trafic d'esclaves n'a jamais été l'exclusif des Européens - une évidence!-, son aspect quantitatif, durable et intégré au développement du système de plantations et à l'accumulation du capital à l'origine de la révolution industrielle en Europe du NordOuest, ont bien été un cas unique dans l'histoire. Les États africains (y compris swahilis dans l'océan Indien) qui se sont lancés dans la traite (et non seulement dans l'esclavage) l'ont fait parce que la demande et le marché existaient: or cette demande et ce marché étaient avant tout européens à partir $\mathrm{du} \mathrm{XVI} \mathrm{I}^{\mathrm{e}}$ siècle. traite comme un " crime contre l'humanité » établirait « de ce fait » une " comparaison » avec un génocide (la Shoah) et il conviendrait donc d'insister sur le fait que les traites ne sont pas un génocide... mais c'est justement ce que ne disait pas la loi Taubira! Alors pourquoi considérer cette loi comme un "problème "? Le fait que la traite ne fut pas un génocide interdit-il de la qualifier de crime contre l'humanité ? De plus, la phrase « La traite n'avait pas pour but d'exterminer un peuple», acceptable sur le plan formel, écartait d'un revers de main la mortalité considérable des esclaves, au plus grand profit des trafiquants appelés à fournir de nouvelles cargaisons. Toute l'économie de l'argumentation la faisait donc comprendre comme une remise en cause des traites en tant que crime contre l'humanité, et donc comme négation de ce qu'affirmait la loi du 23 mai 2001. On peut contester la forme prise par l'émotion du Collectif des Antillais, Guyanais, Réunionnais qui porta plainte pour négation d'un crime contre l'humanité, mais on ne peut pas, suite à de telles formulations, s'étonner de cette émotion comme s'il elle n'avait aucun fondement réel'. Le recours à la polémique soulevée par l'entrevue de o. Pétré-Grenouilleau ne fait donc que brouiller encore plus la critique de l'ouvrage de N. Schmidt.

Enfin, il est toujours désagréable de voir qu'est critiqué comme " politiquement correct » ce que l'on veut dénoncer, le dénonciateur se plaçant ainsi automatiquement du côté de l'insolence salutaire et de l'indépendance critique. Pourtant, jusqu'à il y a peu de temps, c'était la tendance inverse qui était " politiquement correcte " : l'abolitionnisme était une tendance exclusivement européenne (ou nord-américaine). En quoi est-il conformiste de chercher à mieux connaitre la participation des esclaves eux-mêmes, ce à quoi s'essaie, peut-être de manière criticable, N. Schmidt, et à mieux connaitre l'abolitionnisme dans le monde musulman ${ }^{8}$ ? Ce sont des questions que, tout à son vitriol, J.P. Marques ne se pose pas, et on comprend mal, au-delà de critiques qui pourraient être légitimes, le ton cominatoire de son article.

17 - l'article de M. Emilia Madeira SANTos \& Vitor Luís Gaspar RodRIGUES, « No rescaldo da escravatura. As ciências sociais chamadas à liça nos anos 30 (século xx)» (p. 259-273), est d'un style tout autre que le précédent, portant notamment sur la réponse portugaise aux questions du BIT sur le travail forcé, notamment quand Armindo Monteiro était ambassadeur à la SDN. Réflexions intéressantes aussi sur la différence entre esclavage, servitude et travail forcé.

Lusotopie, XV(1) | 2008 
l'esclavage, par José Capela (p. 277-281) et Maciel Santos qui discute le livre de Robert William Fogel, The Slavery Debate. A Retrospective 1952-1990 (Bâton Rouge, Louisiana State University, 2003), qui porte sur les débats dans la presse américaine telle que la New York Review of Books, le New York Times Review of Books, etc. Contact : CEAUP, Via panorâmica, s/n, 4150-564 Porto, Portugal, courriel: <ceaup@letras.up.pt>, site de la revue: $<$ www.africanos.eu/ceaup/>.

\section{NOTES}

1. J'avais déjà signalé ce ton outrancier dans le compte rendu que j'avais fait de son ouvrage Os Sons do silêncio: o Portugal de Oitecentos e a abolição do tráfico de escravos (Lisbonne, ICS, 1999), Lusotopie (Paris, Karthala), 2004 : 432-436. En revanche, sur son nouvel ouvrage, Portugal e as escravatura dos africanos, voir le compte rendu enthousiaste de D. Birmingham dans Lusotopie, XIV (2) : 223-225.

2. En fait, il s'agissait d'ajouter un article 4 à la loi sur les rapatriés du 23 février 2005.

3. Rappelons que cela n'est pas toujours le cas. Puisque Lusotopie est basée à Bordeaux, rappelons par exemple que certains livres de popularisation de l'histoire de cette ville (en texte ou en bande dessinée), taisent totalement la nature négrière du port!

4. Loi $n^{\circ}$ 2001-434 du 23 mai 2001. Voci quelques extraits de cette loi : "Article $1^{\text {er }}$. La République française reconnaît que la traite négrière transatlantique ainsi que la traite dans l'océan Indien d'une part, et l'esclavage d'autre part, perpétrés à partir $\mathrm{du} \mathrm{xv}^{\mathrm{e}}$ siècle, aux Amériques et aux Caraibes, dans l'océan Indien et en Europe contre les populations africaines, amérindiennes, malgaches et indiennes constituent un crime contre l'humanité. Article 2. Les programmes scolaires et les programmes de recherche en histoire et en sciences humaines accorderont à la traite négrière et à l'esclavage la place conséquente qu'ils méritent... ».

5. O. PÉTRÉ-GRENOUILLEAU, Les traites négrières, essai d'histoire globale, Paris, Gallimard, 2004, 474 p., ISBN-13 : 978-2070734993 (« Bibliothèques des histoires »).

6. «Un prix pour Les traites négrières. Interview de O.Pétré-Grenouilleau par Christian Sauvage ", Journal du Dimanche (Paris), 3049, 12 juin 2005.

7. Pour une critique détaillée de cette affaire, on peut se reporter notamment au texte de C. Coquery-Vidrovitch (professeur émérite d'histoire de l'Afrique à l'université de Paris 7), «À propos de l'histoire des traites négrières et, plus généralement, des positions contrastées des Historiens", publié en ligne sur le site du Comité de vigilance face aux usages publics de l'histoire, <http://cvuh.free.fr/spip.php?article64>. Voir aussi, pour une synthèse des débats que le livre lui-même a occasionnés, entre autres, C. CHIVALLON, « Sur une relecture de l'histoire de la traite négrière", Revue d'histoire moderne et contemporaine (Paris, Belin), 2005, LII (4 bis), supplément thématique annuel : 45-53.

8. À ce sujet, voir le récent ouvrage de W.G. CLARENCE-SMITH, Islam and the Abolition of Slavery, Londres, C. Hurst, 2005, 300 p., ISBN-13 : 978-1850657088. 